\title{
Editorial
}

\section{Aktuelle Aspekte des Mammakarzinoms: Teil 2}

\section{Edgar Petru}

Klinische Abteilung für Gynäkologie, Universitätsklinik für Frauenheilkunde und Geburtshilfe, Medizinische Universität Graz, Graz, Österreich

Vor wenigen Monaten ist bereits Teil 1 der "Aktuellen Aspekte des Mammakarzinoms" in der WMW erschienen (Wien Med Wochenschr 2010:160/ 7-8). Im Teil 2 werden acht zusätzliche wichtige Teilbereiche dieser Tumorentität unter Berücksichtigung verschiedenster Disziplinen behandelt.

Der Themenbereich „Vererbbarer Brustkrebs jenseits von BRCA1- und BRCA2-Genmutationen" gibt Einblick in einen klinisch zunehmend wichtigen Bereich, nämlich die Situation des Vorgehens bei Patientinnen mit belastender Familienanamnese, bei denen jedoch die Testung auf die beiden wichtigsten Tumorsuppressorgene BRCAl und 2 negativ ist. Hier sind in den letzten Jahren große Fortschritte erzielt worden.

Die „hormonelle Kontrazeption und das Risiko, an Brustkrebs zu erkranken", ist ein Themenbereich, der nahezu jede Frau betrifft. Die von Frau Bjelic-Radisic dargestellte Übersicht fasst den derzeitigen Wissensstand zusammen. Wertvoll erscheint die klare Unterscheidung in Östrogen-Gestagenkombinationen und rein gestagenbasierte Kontrazeptionsmaßnahmen.

„Fertilitätserhaltende Maßnahmen und die Behandlung der Infertilität bei Brustkrebspatientinnen" ist ein Themenbereich, der auch immer mehr an Bedeutung gewinnt. Dies v. a. deshalb, da sich generell in der Bevölkerung der Wunsch, Kinder zu bekommen, in die späteren Lebensjahre verschoben hat. Frauen mit Brustkrebs weisen heute eine bessere Prognose auf. Somit gilt der verlagerte Kinderwunsch ganz besonders auch für junge Patientinnen nach Brustkrebs.
„Onkoplastische und rekonstruktive Operationen der Brust" stellen in jedem Alter im Gesamtbetreuungskonzept von Frauen mit Brustkrebs einen essentiellen Bestandteil dar. Sie werden auch illustrativ von Frau Gschwantler-Kaulich beleuchtet.

Einen weiteren operativen Schwerpunkt stellt die Wächterlymphknotenbiopsie dar, die mittlerweile die Therapie des Mammakarzinoms maßgeblich verändert hat. Mit deren Hilfe ist es heute möglich, bei vielen Patientinnen die Entwicklung eines klinisch relevanten Lymphödems zu verhindern.

Die Grundlagen und Wirkungsweise einer zielgerichteten Therapie bei Brustkrebs werden von Herrn Singer dargestellt, von Herrn Hubalek die Mechanismen der Resistenzentwicklung gegenüber Trastuzumab sowie die Strategien zu deren Überwindung. Schließlich wird auch der Aspekt einer komplementären Therapie mit proteolytischen Enzymen in einem Beitrag behandelt. Er weist auf die Bedeutung von Studien gerade in diesem wissenschaftlich interessanten Feld hin.

Wir hoffen sehr, dass auch die ausgewählten Themen in diesem 2. Themenschwerpunkt das Interesse der Leserschaft der WMW wecken werden und einen Überblick über interessante aktuelle Entwicklungen bieten können.

\section{Interessenskonflikt}

Es besteht kein Interessenskonflikt. 